\title{
QCD Physics in Atlas at the Large Hadron Collider
}

\author{
James Proudfoot ${ }^{*}$ \\ Argonne National Laboratory, High Energy Physics Division, \\ 9700 S. Cass Avenue, Argonne Illinois, 60439 USA
}

\section{INTRODUCTION}

The Large Hadron Collider (LHC) is a protonproton collider with a $14 \mathrm{TeV}$ center of mass energy. The design luminosity is $10^{34} \mathrm{~cm}^{-1} \mathrm{~s}^{-1}$ with beam collisions separated by $25 \mathrm{~ns}$. The initial operation for physics will take place at a luminosity of $10^{33} \mathrm{~cm}^{-1} \mathrm{~s}^{-1}$ and it is expected that the integrated luminosity delivered in the first year will be $10 \mathrm{fb}^{-1}$. This integrated luminosity will result in very large event samples for most processes, for example: $\sim 10^{8}$ leptonic $\mathrm{W}$ decays, $10^{4} \gamma$ 's with $\mathrm{E}_{\mathrm{t}}>500 \mathrm{GeV}$ and $10^{4}$ jets with $E_{t}>1 \mathrm{TeV}$. As a result of the high statistics event samples, the understanding of most QCD processes at $14 \mathrm{TeV}$ will be systematics limited after the first year of running.

The Atlas detector [1] is a general purpose detector designed to be sensitive to the many physics processes which are expected at the LHC. It contains high performance tracking using silicon detectors and a transition radiation tracker in a 2 Tesla solenoidal magnetic field, a high resolution electromagnetic calorimeter based on lead-liquid argon, a hadron calorimeter based on steel-scintillator and $\mathrm{Cu} / \mathrm{W}$ liquid argon, and a large instrumented air-core toroid magnet system for muon measurement. The basic performance characteristics of these systems are given in Table 1.

\section{THE KINEMATIC REACH OF THE LHC}

The increased kinematic reach of the LHC is indicated in Fig. 1 [2], which compares the jet inclusive cross-section at the Fermilab Tevatron with

\footnotetext{
*Work supported in part by the US Department of Energy, High Energy Physics Division, under Contract W-31-109Eng-38.
}

\begin{tabular}{|l|l|}
\hline Detector Sub-System & Performance \\
\hline $\begin{array}{l}\text { Tracking (Si + Transition } \\
\text { Radiation Tracker) }\end{array}$ & $\sigma / \mathrm{p}_{\mathrm{t}} \sim 5.10^{-4} \mathrm{p}_{\mathrm{t}}(\mathrm{GeV})+0.01$ \\
\hline $\begin{array}{l}\text { EM Calorimeter }(\mathrm{Pb} \\
\text { Liquid Argon) }\end{array}$ & $\sigma / \mathrm{E} \sim 10 \% / \sqrt{E}(\mathrm{GeV})$ \\
\hline $\begin{array}{l}\text { Hadron Barrel } \\
\text { Calorimeter (Steel }+ \\
\text { Scintillator) }\end{array}$ & $\sigma / \mathrm{E} \sim 50 \% / \sqrt{E}(\mathrm{GeV})+0.03$ \\
\hline $\begin{array}{l}\text { Hadron Endcap } \\
\text { Calorimeter }(\mathrm{Cu} / \mathrm{W}+ \\
\text { Liquid Argon) }\end{array}$ & $\sigma / \mathrm{E} \sim 60 \% / \sqrt{E}(\mathrm{GeV})+0.03$ \\
\hline $\begin{array}{l}\text { Muon (Instrumented Air } \\
\text { Toroid) }\end{array}$ & $\sigma / \mathrm{p}_{\mathrm{t}} \sim 10 \%$ at $1 \mathrm{TeV}$ \\
\hline
\end{tabular}

Table 1. Basic performance characteristics of the Atlas detector for the $\mathrm{LHC}$.

the LHC. The increased reach in $\mathrm{E}_{\mathrm{t}}$ is almost a factor of 10. QCD phenomenology can make predictions for inclusive jet cross-sections as well as other observables in this unexplored region of $\mathrm{x}$ and $\mathrm{Q}^{2}$ and $\mathrm{E}_{\mathrm{t}}$. The overall consistency with QCD will provide an extensive test of the predictive power of this theory. In addition, QCD processes form the majority of the background processes in many of the searches, which will be carried out at the LHC. Some expectations for QCD physics with the Atlas detector are discussed below. These and other physics issues are discussed in more detail in reference [3].

\section{3. “ENGINEERING” ISSUES}

Many detector response and physics characteristics impact the overall performance of the Atlas detector for QCD physics. One physics issue is shown in Fig. 2, which shows the Monte Carlo predictions [3] of several models for the charged particle multiplicity in 


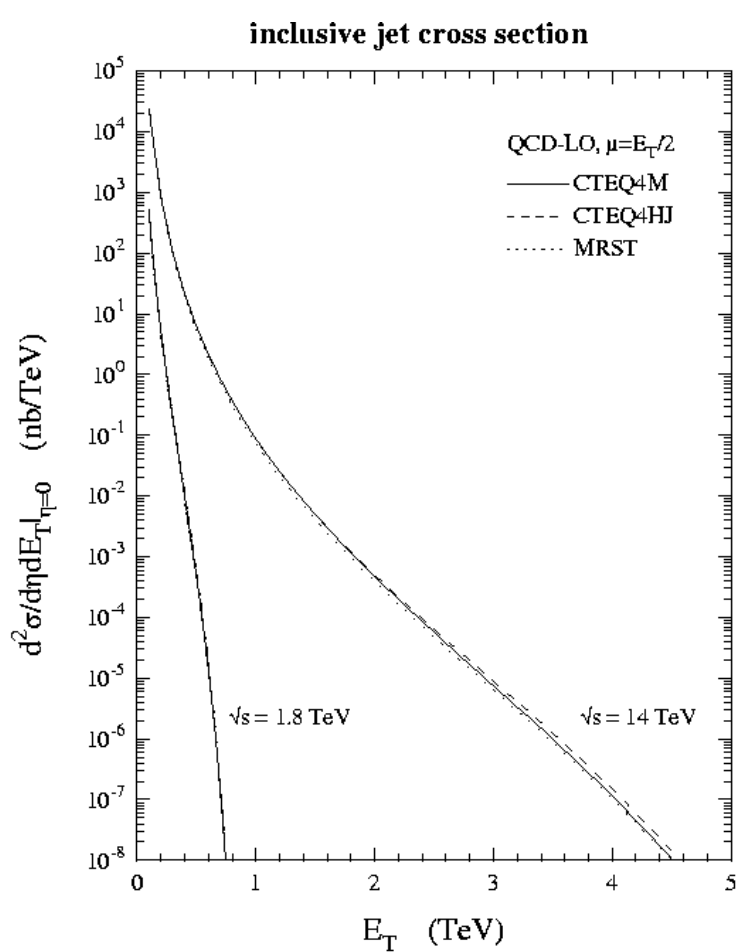

Fig. 1. Jet inclusive cross-section at the Tevatron compared to the predicted cross-section for the LHC.

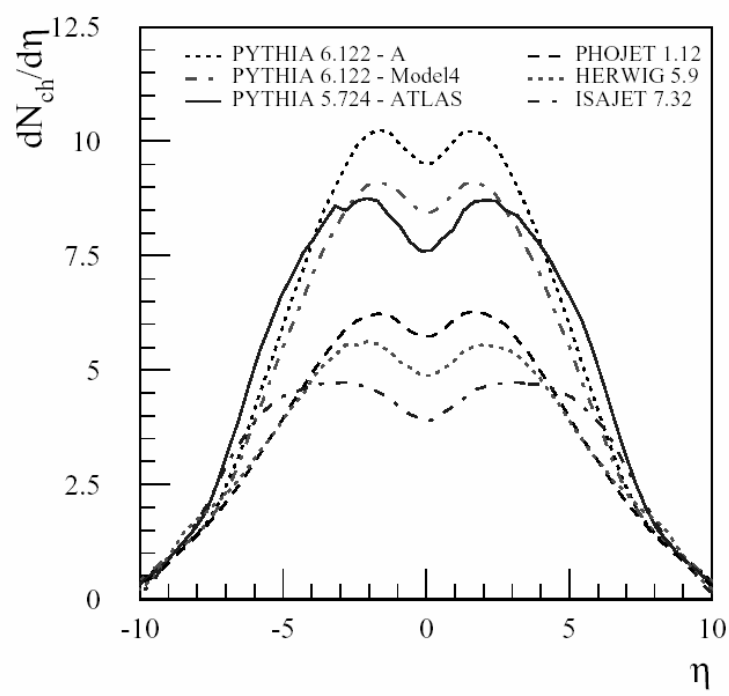

Figure 2. Monte Carlo predictions for charged particle multiplicity distributions, for several models as described in [3]. minimum bias events. The prediction varies from 5 to 10 particles per unit rapidity in the central region. The underlying event, resulting from overlapping minimum bias events, is one of the energy corrections in jet measurement contributing significantly to jet $E_{t}$ systematics. Therefore, this measurement is both an interesting test of (nonperturbative) QCD which is accessible at low luminosity, and a necessary "engineering" measurement for jet reconstruction.

A Geant simulation of the calorimeter predicts a jet energy resolution of $\sim 2 \%$ for high $\mathrm{E}_{\mathrm{t}}$ jets. There are many systematics to jet measurement including: the jet energy scale which is expected to be $\sim 1 \%$ from in situ calibration; calorimeter non-linearity $(<3 \%)$; and effects such as the jet reconstruction algorithm used and energy from the underlying event. These are discussed in detail in the Atlas Physics TDR [3].

\section{DETERMINATION OF PARTON DISTRIBUTION FUNCTIONS (PDF's)}

In QCD phenomenology, the production crosssection for any process is factorized into the product of the parton-parton cross-section and the parton distribution function (or pdf). These pdf's are determined by fitting the cross-sections for several processes and from many experiments. The sensitivity of the jet $\mathrm{E}_{\mathrm{T}}$ cross-section to different sets of pdf's was studied in the Atlas Physics TDR [3]. At high $\mathrm{E}_{\mathrm{T}}$, the variation in the cross-section is $\sim 20 \%$. Once data from the LHC is available, the pdf's will also be directly measured by including results from the LHC in the global fits. For example, inclusive photon production provides input to the gluon distribution function since the two most important parton level processes are $q g \rightarrow q \gamma$ and $q q \rightarrow g \gamma$. Measurements of photons with $\mathrm{E}_{\mathrm{t}}$ from $40-500 \mathrm{GeV}$ will provide information on the gluon density for $5.10^{-4}<x<0.2$. Another good example of sensitivity to pdf's is the asymmetry in $\mathrm{W}^{+} v s . \mathrm{W}^{-}$production at high rapidity [3], as shown in Fig. 3. At high rapidity, $\mathrm{W}$ production is dominated by valence quark annihilation with sea quarks and therefore, the $\mathrm{W}^{+}$ production cross-section is larger than that for $\mathrm{W}^{-}$. This process will provide sensitivity to both sea and valence quarks with $\mathrm{x}_{1} \mathrm{x}_{2} \sim 3 \times 10^{-5}$. 


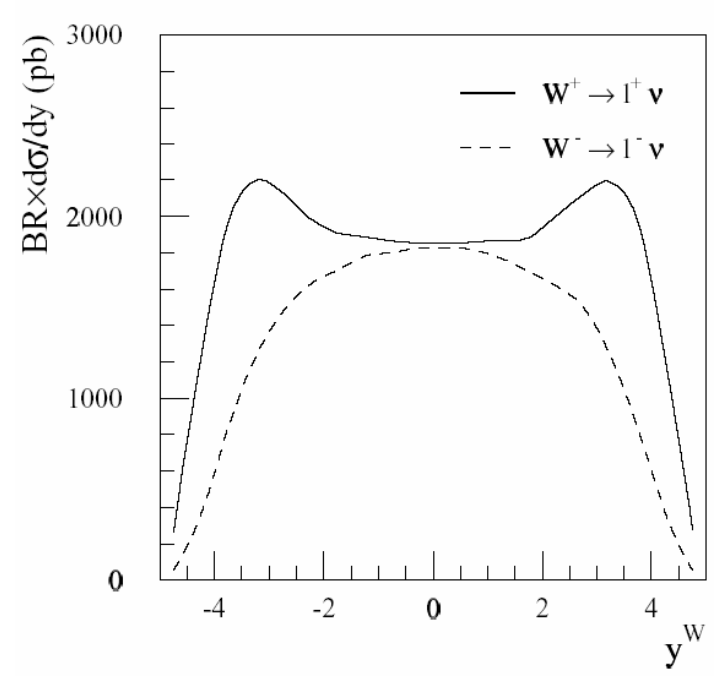

Figure 3. Pseudo-rapidity distributions for $\mathrm{W}^{+}$ and $\mathrm{W}^{-}$production in pp collisions at $14 \mathrm{TeV}$.

\section{MEASUREMENT OF THE STRONG COUPLING CONSTANT, $\alpha_{\mathrm{s}}$.}

In general there can be a strong correlation between the value of $\alpha_{s}$ and fitted pdf's, in the results from global fits. A study was carried out by Atlas to evaluate a method which allows the possibility of decoupling these two measurements [4]. The approach taken is to fit the inclusive jet cross-section as a function of $E_{t}$ and $\alpha_{s}\left(E_{t}\right)$ in two bins of pseudorapidity and study its sensitivity to different sets of pdf's. By inverting this fit, one can then determine $\alpha_{s}\left(E_{t}\right)$. An example of this analysis is shown in Fig. 4. The results indicate that $\alpha_{\mathrm{s}}$ can be determined with an overall precision of $\sim 10 \%$, in which the contribution to the uncertainty from our knowledge of pdf's is $\sim 3 \%$.

\section{SUMMARY}

The LHC will provide a unique opportunity to test the predictive power of $\mathrm{QCD}$, probing regions in $\mathrm{x}$ and $\mathrm{Q}^{2}$ well beyond those studied to date. Almost any process one can think of will contribute to this understanding: from a measurement of the total cross section and inclusive cross sections of b's, t's, and W's to searches for physics beyond the Standard Model. In addition, a measurement of $\alpha_{s}\left(E_{t}\right)$ out to $E_{t}$

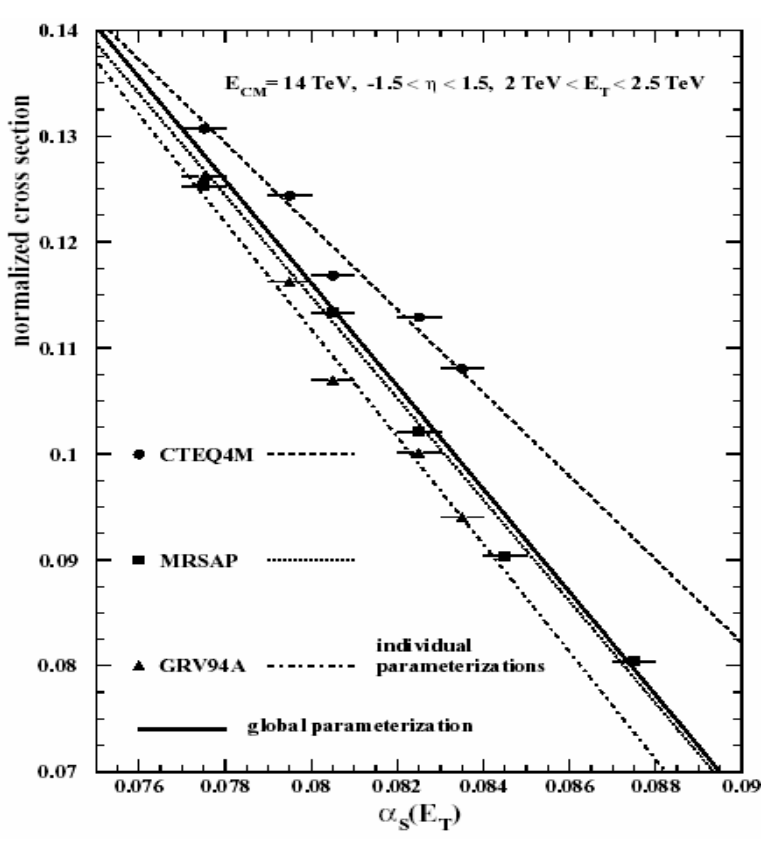

Figure 4. An example fit of $\alpha_{s}\left(E_{t}\right)$ used in the determination of the precision with which $\alpha_{\mathrm{s}}$ may be measured at the LHC.

$\sim 3 \mathrm{TeV}$ with a precision of $\sim 10 \%$ should be possible.

\section{ACKNOWLEDGMENTS}

I could not have prepared this presentation without the work of my colleagues in the Atlas physics groups and the many contributors to the Atlas Physics Technical Design Report. For this I give my thanks. I would like to thank, in particular, Fabiola Gianotti, Stefan Tapprogge, Joey Huston, and Bob Blair for informative discussions on QCD physics.

\section{REFERENCES}

1. ATLAS Technical Proposal, CERN/LHCC 94-43.

2. J. Huston, private communication.

3. ATLAS Detector and Physics Performance Technical Design Report, CERN/LHCC 99-14 and 99-15 (1999).

4. Determination of $\alpha_{s}$ using jet cross-section parameterizations at hadron colliders, H. Stenzel, ATL-PHYS-2001-003. 